\title{
Azione detossicante dell'acqua minerale Rocchetta
}

\author{
N. Di Paolo, D. Sassone, F. Cappelletti, P. Rossi, G. Garosi \\ U.O. Nefrologia e Dialisi \\ Azienda Ospedaliera Senese
}

e acque minerali sono acque sorgive che risultano dotate di particolari proprietà biologiche e terapeutiche, da sempre utilizzate dall'uomo nella cura delle affezioni più varie $(1,2)$.

La loro origine è atmosferica: cadono sulla superficie terrestre come precipitazioni e penetrano più o meno profondamente nel sottosuolo, a seconda della costituzione e disposizione degli strati che incontrano; dopo un percorso variabile, si riuniscono in falde acquee che affiorano alla superficie. Durante questo percorso, le acque vengono a contatto con formazioni rocciose a opera delle quali vengono sottoposte a processi di filtrazione che le caratterizzano da un punto di vista fisico-chimico (3).

Già dai tempi dei Normanni erano note le capacità depurative della fonte Rocchetta, sita sulle montagne dell'Appennino Umbro-Marchigiano intorno a Gualdo Tadino: questa fonte ci dona un'acqua oligominerale con un discreto contenuto di bicarbonato e calcio e arricchita da numerosi oligoelementi caratteristici della formazione geologica dell'area Rocchetta (4).

Secondo recenti indicazioni (5), un'acqua è oligominerale se il tenore dei sali minerali in essa contenuti, calcolato come Residuo Fisso, non è superiore a $500 \mathrm{mg} / \mathrm{L}$ : l'acqua Rocchetta ha un Residuo Fisso di $173.06 \mathrm{mg} / \mathrm{L}$. Questo tipo di acqua minerale è tradizionalmente utilizzata nelle cure idropiniche: essa viene rapidamente assorbita dall'apparato digerente (in buona quantità, già dallo stomaco) e ha capacità di promuovere un rapido incremento del volume extracellulare e del flusso ematico renale: ne risulta una poliuria a basso peso molecolare che aumenta il volume urinario e sottrae numerosi cataboliti litogeni ai processi di nucleazione o di neoapposizione a nuclei già costituiti, che sono precursori della concrezione litiasica renale (6); in realtà i benefici che si traggono dall'assunzione di un'acqua oligominerale, qual è la Rocchetta, la pongono al centro dell'attenzione negli studi sul ruolo terapeutico delle acque oligometalliche nelle affezioni epatiche, del metabolismo, del sistema cardiovascolare oltre che nelle affezioni di interesse nefro-urologico.

In altre parole non si riesce ancora a trovare una spiegazione scientifica razionale che sia in grado di chiarire il fenomeno universale del ricorso alle facoltà curative delle acque, feno- meno che la moderna medicina, così capace di distruggere qualsiasi terapia non ortodossa, non è riuscita minimamente a scalfire, anzi in tutto il mondo sia la terapia termale che quella idropinica sono in continua espansione (6).

In ogni caso negli ultimi decenni anche la letteratura internazionale si sta dedicando allo studio degli effetti farmacologici che le acque minerali esercitano sull'organismo e alcune questioni si cominciano per lo meno ad affrontare con quel rigore scientifico che la moderna medicina impone. Con il presente studio abbiamo testato la rivendicata efficacia depurativa dell'acqua oligominerale Rocchetta, confrontandone gli effetti con quelli di un'acqua di conduttura, scelta a caso in un'area dell'hinterland pisano.

\section{Materiali e metodi}

La sperimentazione si è basata essenzialmente su di un carico idrico consistente nell'assunzione di $20 \mathrm{ml} / \mathrm{kg}$ di peso corporeo, assunti in un tempo di 30 minuti.

La Ditta Rocchetta ha fornito 16 bottiglie di 2 litri cadauna con una sola etichetta con numerazione progressi- 


\section{TABELLA I - CARATTERISTICHE CHIMICO-FISICHE DELL'ACQUA DI CONTROLLO}

\begin{tabular}{lc}
\hline Conducibilità a $20^{\circ}(\mu \mathrm{S} / \mathrm{cm})$ & 823 \\
$\mathrm{pH}$ & 6.9 \\
Ione $\mathrm{Ca}(\mathrm{mg} / \mathrm{L})$ & 114.4 \\
Ione $\mathrm{Na}(\mathrm{mg} / \mathrm{L})$ & 72.1 \\
Ione $\mathrm{Mg}(\mathrm{mg} / \mathrm{L})$ & 18.4 \\
Ione $\mathrm{K}(\mathrm{mg} / \mathrm{L})$ & 1.1 \\
Ione $\mathrm{HCO}_{3}(\mathrm{mg} / \mathrm{L})$ & 443.2 \\
Ione solforico $(\mathrm{mg} / \mathrm{L})$ & 46 \\
Ione cloridrico $(\mathrm{mg} / \mathrm{L})$ & 67.8 \\
Ione nitrico $(\mathrm{mg} / \mathrm{L})$ & 7 \\
Ione fluoridrico $(\mathrm{mg} / \mathrm{L})$ & non rilevato \\
Ione ioduro $(\mathrm{mg} / \mathrm{L})$ & non rilevato \\
Ione ammonio $(\mathrm{mg} / \mathrm{L})$ & non rilevato \\
Ione nitroso $(\mathrm{mg} / \mathrm{L})$ & non rilevato \\
$\mathrm{Fe}$ & assente \\
$\mathrm{Cl}$ & assente \\
\hline
\end{tabular}

\section{TABELLA II - CARATTERISTICHE CHIMICO-FISICHE DELL'ACQUA ROCCHETTA}

\begin{tabular}{lc}
\hline Conducibilità a $20^{\circ}(\mu \mathrm{S} / \mathrm{cm})$ & 315 \\
$\mathrm{pH}$ & 7.79 \\
Ione $\mathrm{Ca}(\mathrm{mg} / \mathrm{L})$ & 57 \\
Ione $\mathrm{Na}(\mathrm{mg} / \mathrm{L})$ & 4.6 \\
Ione $\mathrm{Mg}(\mathrm{mg} / \mathrm{L})$ & 3.7 \\
Ione $\mathrm{K}(\mathrm{mg} / \mathrm{L})$ & 0.46 \\
Ione $\mathrm{HCO}_{3}(\mathrm{mg} / \mathrm{L})$ & 180 \\
Ione solforico $(\mathrm{mg} / \mathrm{L})$ & 8.5 \\
Ione cloridrico $(\mathrm{mg} / \mathrm{L})$ & 7.7 \\
Ione nitrico $(\mathrm{mg} / \mathrm{L})$ & 1.2 \\
Ione fluoridrico $(\mathrm{mg} / \mathrm{L})$ & 0.14 \\
Ione ioduro $(\mathrm{mg} / \mathrm{L})$ & non rilevato \\
Ione ammonio $(\mathrm{mg} / \mathrm{L})$ & 0.19 \\
Ione nitroso $(\mathrm{mg} / \mathrm{L})$ & non rilevato \\
$\mathrm{Fe}$ & non rilevato \\
$\mathrm{Cl}$ & non rilevato \\
\hline
\end{tabular}

va da 1 a 16: 8 contenevano 2 litri di acqua Rocchetta e le altre 8 di un'acqua di un acquedotto della Provincia di Pisa (Tabb. I e II).

Né gli sperimentatori, né i soggetti in esame erano a conoscenza del tipo di acqua contenuto nelle bottiglie.

L'analisi della conducibilità, del contenuto sodico e del $\mathrm{pH}$ delle due acque rende ragione dell'origine sorgiva e delle proprietà oligometalliche dell'acqua Rocchetta (Fig. 1).

Dopo averli ampiamente edotti sullo scopo della sperimentazione e ottenuto il loro consenso, abbiamo chiesto a 16 soggetti di età compresa tra i 28 e i 52 anni (età media $35 \pm 1.4$ anni, 10 ma- schi e 6 femmine volontari e in perfetta salute, scelti a caso in una popolazione studentesca e infermieristica) di osservare una dieta consistente in $1 \mathrm{~g}$ di proteine e 30 calorie/ $\mathrm{kg}$ di peso corporeo, nei 4 giorni precedenti la prova. La prova consisteva nel carico idrico realizzato al mattino presto, su soggetti digiuni e dopo averli fatti urinare (8 hanno assunto acqua Rocchetta ed 8 acqua di controllo); dopo la minzione e prima del carico idrico, è stata effettuata un'ecotomografia vescicale che ci ha permesso di sincerarci del completo svuotamento della vescica e dell'assenza di residuo postminzionale.
Nelle 4 ore successive al carico idrico, sono state raccolte le urine e i campioni di sangue e, alla fine della quarta ora, è stata realizzata una nuova valutazione ecografica vescicale al fine di accertarci del completo svuotamento vescicale; a intervalli orari, è stata valutata la pressione arteriosa.

Sui campioni raccolti, sono state effettuate le seguenti rilevazioni:

- Misurazione della diuresi

- pH urinario

- Peso specifico

- Azoturia

- Creatininuria

- Clearance dell'urea

- Clearance della creatinina

- Clearance dell'acido urico

- Clearance dei fosfati

- Calciuria

- Ossaluria

- Natriuria

- Potassiuria

La sperimentazione è stata effettuata con il consenso del Comitato Etico Locale e secondo le norme dettate da Helsinki.

I dati ottenuti sono stati elaborati statisticamente utilizzando il MannWhitney-Wilcoxon test.

\section{Risultati}

Non abbiamo rilevato effetti collaterali del carico idrico e variazioni della pressione arteriosa rispetto ai valori basali in nessuno dei soggetti in esame.

Non esistono differenze significative tra le due acque alla valutazione del $\mathrm{pH}$ urinario, del peso specifico urinario, della clearance dei fosfati, della calciuria, ossaluria, natriuria, potassiuria e della quantità della diuresi.

Con notevole sorpresa, abbiamo constatato che la media della clearance della creatinina $(92 \pm 2.68$ $\mathrm{ml} / \mathrm{min}$ ), della clearance dell'acido urico $(7 \pm 1.84 \mathrm{ml} / \mathrm{min})$ e della clearance dell'urea $(41.8 \pm 7.51 \mathrm{ml} / \mathrm{min})$ dei pazienti che hanno assunto acqua Rocchetta risultano maggiori rispetto ai valori delle stesse clearances presentate dal gruppo di controllo $(60.5 \pm 20.1 \mathrm{ml} / \mathrm{min} ; 5 \pm 1.4$ $\mathrm{ml} / \mathrm{min} ; 28.8 \pm 7.1 \mathrm{ml} / \mathrm{min}$ rispettivamente) (Figg. 2, 3, 4). 
Anche l'escrezione di creatinina, urato e urea sono risultate maggiori nel gruppo che ha assunto acqua Rocchetta rispetto al gruppo di controllo (Figg. 5, 6, 7).

\section{Discussione}

Sebbene sia difficile verificare l'effetto terapeutico di un'acqua minerale, esso è tradizionalmente riferito alla capacità che tale acqua possiede di promuovere la diuresi: questo effetto incrementerebbe i flussi urinari e, sottraendo cataboliti litogeni e promuovendone l'escrezione, contrasterebbe gli eventi nucleativi e la neoapposizione a nuclei già costituiti di materiali organici e inorganici (6); inoltre l'aumento dei flussi urinari contrasterebbe la stasi urinaria, fattore favorente le flogosi infettive che spesso complicano la patologia litiasica (7).

L'uomo ha una diuresi media di 800$1000 \mathrm{ml} /$ die che corrisponde a un volume di 0.55-0.96 ml/min che, se considerato sui due ureteri, ci darà un

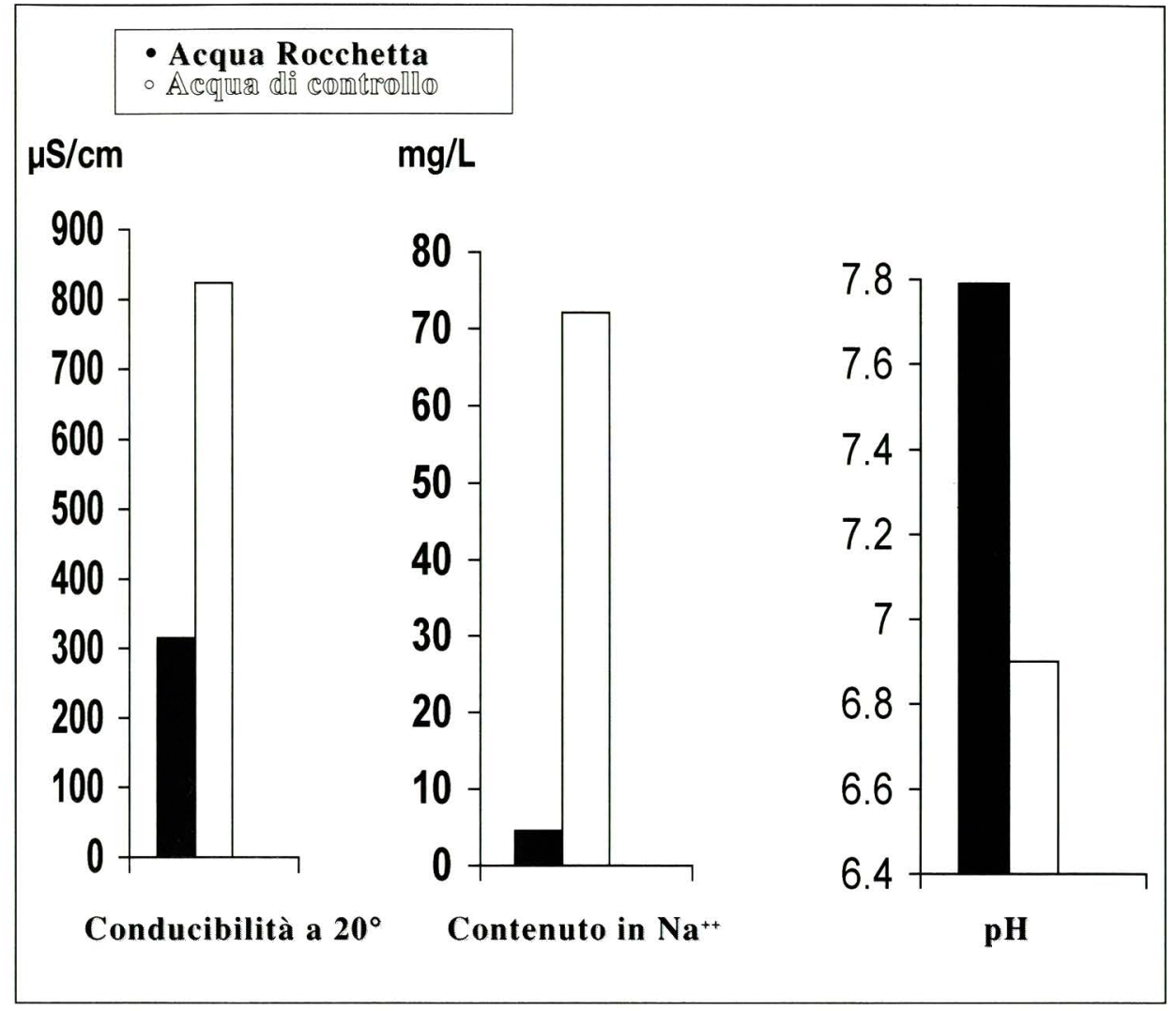

Fig. 1 - Confronto tra conducibilità, contenuto sodico e pH delle due acque in esame.

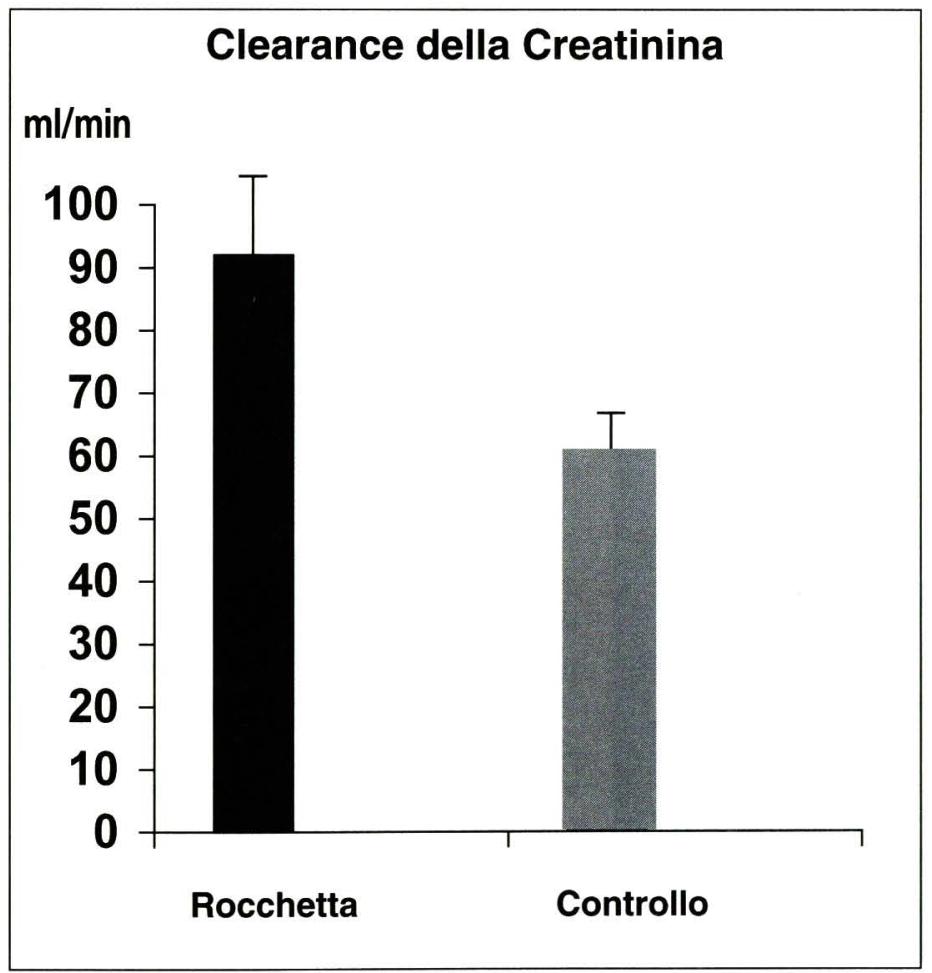

Fig. 2 - Comportamento della clearance della creatinina dopo carico idrico con acqua Rocchetta e acqua di controllo.

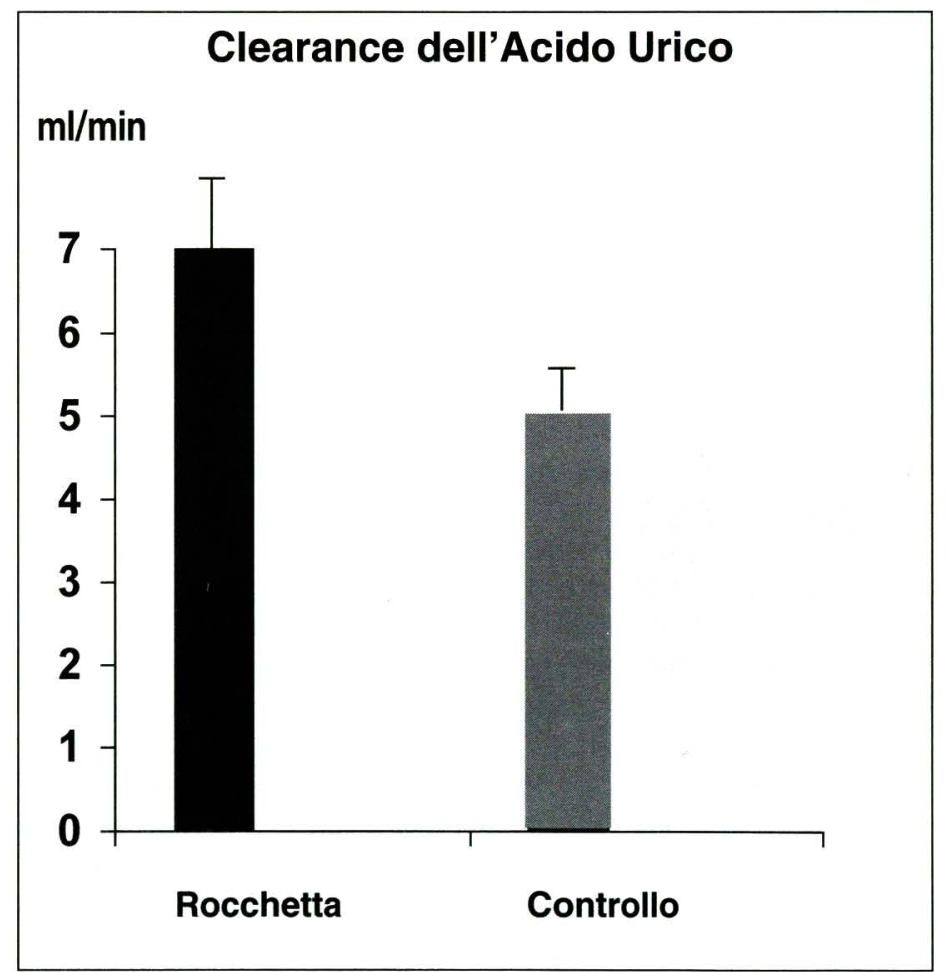

Fig. 3 - Comportamento dell' acido urico dopo carico idrico con acqua Rocchetta e acqua di controllo. 


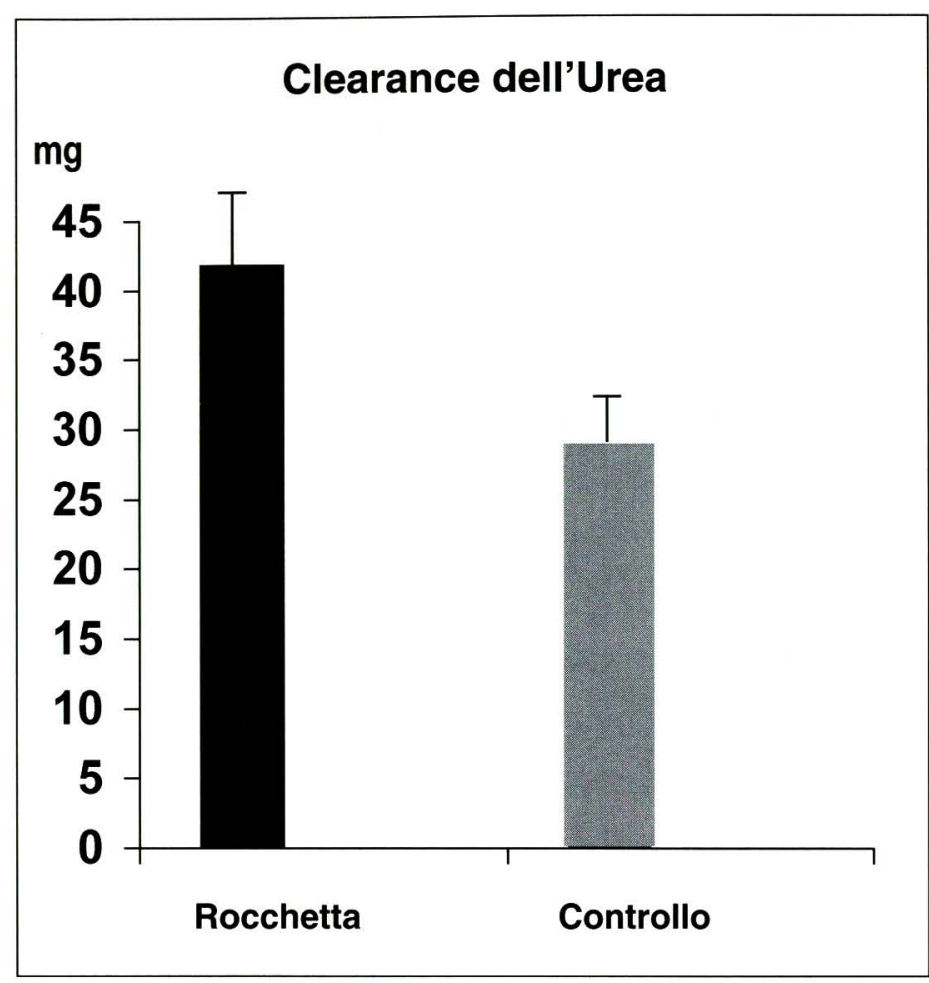

Fig. 4 - Comportamento della clearance dell' urea dopo carico idrico con acqua Rocchetta e acqua di controllo.

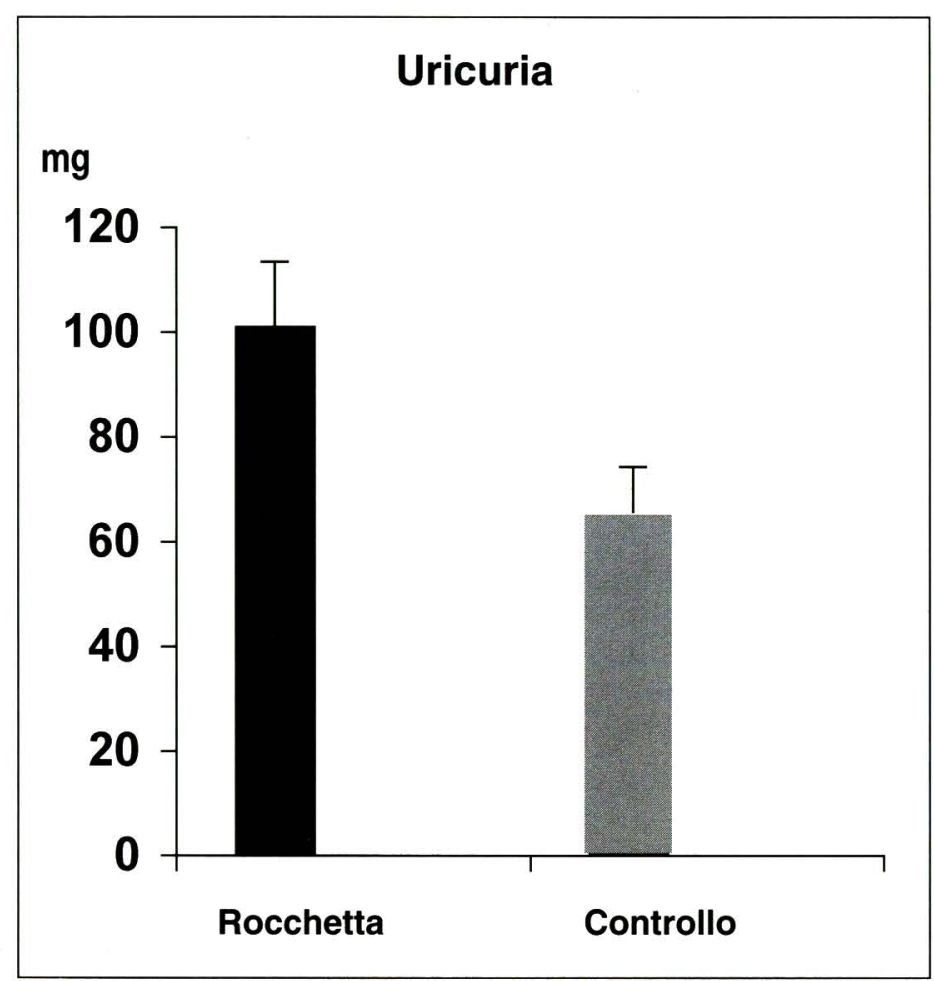

Fig. 6 - Comportamento dell' uricuria dopo carico idrico con acqua Rocchetta e acqua di controllo.

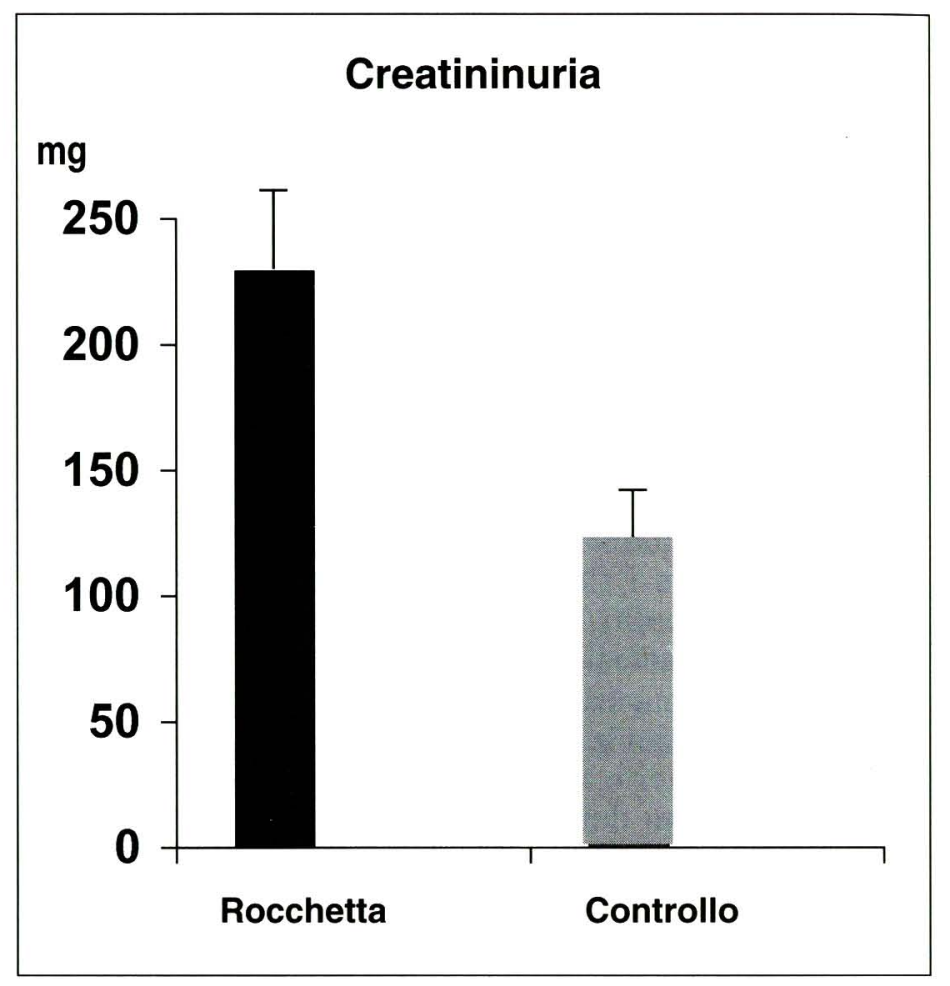

Fig. 5 - Comportamento della creatininuria dopo carico idrico con acqua Rocchetta e acqua di controllo.

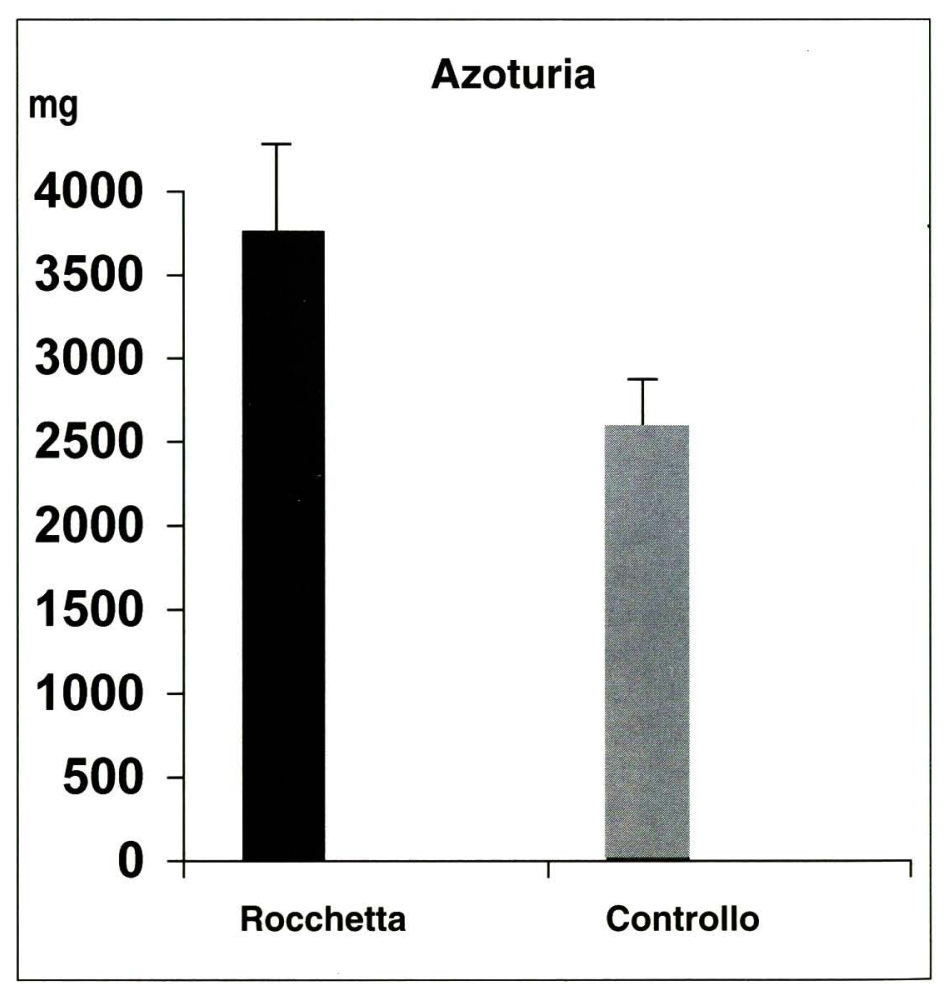

Fig. 7 - Comportamento dell' azoturia dopo carico idrico con acqua Rocchetta e acqua di controllo. 
effetto di transito di tipo capillare. Se aumentiamo la diuresi di 3 volte (3 litri/die) otteniamo un volume-minuto di $1.66-2.08 \mathrm{ml}$; se l'introduzione dei liquidi si effettua in sole due ore si potrà ottenere, nelle ore successive l'assunzione, un volume-minuto di $25 \mathrm{ml}(12.5 \mathrm{ml} / \mathrm{min}$ per distretto).

Il concetto per cui questa condizione è utile nella terapia e prevenzione della litiasi renale è stato supportato per secoli senza dimostrazioni scientifiche corrette: esistono infatti pochi studi prospettici sulla terapia idropinica nella nefrolitiasi (8-10); questi Autori hanno dimostrato che i pazienti con nefrolitiasi calcica idiopatica possono prevenire la recidiva delle calcolosi con il solo aumento della diuresi e senza modificazioni della dieta, con l'esclusione degli ipercalciurici nei quali è indispensabile ridurre il calcio urinario con la dieta o con la terapia farmacologica. Nel 1988 la Consensus Conference (11) ritenne importante consigliare ai pazienti litiasici renali di incrementare la diuresi portandola al di sopra di 2 litri/die. D'altra parte molti Autori hanno evidenziato come particolari condizioni climatiche e ambientali, che provocano disidratazione, possano favorire la formazione di calcoli renali (12-16). Altro dato degno di essere ricordato è che numerosi studi di fisiologia delle vie escretrici (17) hanno dimostrato che il carico idrico comporta, a livello ureterale, un aumento vivace della peristalsi, sia come numero che come durata di contrazioni (è probabile che esistano, nel bacinetto renale, osmo e barocettori) e come questo sia causa di un aumento della pressione intraureterale (da valori medi di 5-15 a 50-80 $\mathrm{cmH}_{2} \mathrm{O}$ ): questo fenomeno sarebbe alla base dell'incremento della forza di trasporto e di erosione delle urine che "detergerebbe" il tratto urinario dai nuclei concretivi destinati a aumentare di volume. La diuresi da acque minerali dovrebbe, per ottenere gli effetti appena elencati, essere caratterizzata da un'eliminazione di urine superiore in quantità all'acqua ingerita, eliminazione di sodio e di altri oligoelementi, risposta pronta (già dopo 2-3 ore dall'assunzione): queste risposte non corrispondono sempre all'ipotonia del liquido ingerito (18).

Infatti il principio secondo il quale le acque oligominerali vanno considerate principalmente per il loro effetto diuretico, perde sempre più valore: l'importanza di altri probabili meccanismi d'azione che per il passato erano stati supposti solo in via del tutto teorica, si fa sempre più evidente (19). Infatti non è sempre vero che le acque oligominerali sono fortemente diuretiche e che a tale attività va riferita gran parte dell'azione terapeutica.

Alcuni studi italiani sulle acque oligominerali (20) a basso residuo fisso hanno evidenziato come la loro capacità di promuovere la diuresi non sia particolarmente elevata rispetto ad altre acque: di contro altri autori hanno notato perfino una maggiore diuresi, come risultato dell'assunzione di acqua di fonte rispetto al carico idrico praticato con acque oligominerali $(21,22)$.

Tali studi sono stati condotti con la metodica del carico idrico e valutazione della risposta diuretica nelle ore immediatamente successive: i risultati di queste prove potrebbero essere ritenuti dubbi sulla base dell'osservazione fatta da alcuni $(20,22)$ che l'apparato renale, non allenato allo stimolo rappresentato dal carico idrico, possa reagire a esso in modo tale da fornire dati non corrispondenti alla reale capacità di stimolo della diuresi che l'acqua in esame è in grado di esercitare. Tuttavia questa ipotesi è poco accettabile se si pensa al grande utilizzo che si è fatto fino a poche decine di anni or sono della prova di diluizione che in base a precisi principi di fisiologia renale ha dato un contributo positivo alle indagini di semeiotica renale (23).

Sempre a tale proposito alcuni autori (20) hanno protratto lo stimolo della terapia idropinica e la relativa osservazione per diversi giorni: essi non hanno osservato un incremento significativo della diuresi rispetto al gruppo di controllo, ma piuttosto una risposta "metabolica" caratterizzata da una notevole natriuresi, scomparsa di edemi e attenuazione dell'iperemesi in pazienti in gravidanza.
D' altra parte è estremamente difficile verificare l'effetto depurativo di un'acqua minerale.

La letteratura inerente l'azione depurativa delle acque minerali è ricca di lavori effettuati in tutto il mondo, ma di scarso valore scientifico in quanto mancano di una revisione critica e la loro attendibilità è estremamente discutibile in quanto pubblicate in riviste nazionali, mentre la letteratura internazionale qualificata ignora, o quasi, tale tipo di contributi. Tutto ciò scaturisce dal fatto che è molto problematico dimostrare un'azione favorente l'eliminazione da parte dell'organismo di tossici endogeni da parte di un'acqua minerale. D'altra parte quest'azione detossicante viene rivendicata da varie acque minerali: recenti contributi sperimentali di autori russi (24) hanno evidenziato sia nell'animale che nell'uomo la capacità di alcune acque oligominerali (Satanovka, Naftus, Tib2, Celkarskaria e altre) di promuovere un effetto depurativo caratterizzato dall'escrezione di urea e acido urico dovuto, secondo gli autori, alla riduzione del loro riassorbimento tubulare (25); la conseguente minore presenza di urea nell'interstizio midollare sarebbe la causa della riduzione del potere di concentrazione delle urine (26).

Sull'effetto metabolico delle acque oligominerali esistono invece molti contributi con particolare riguardo agli effetti sul catabolismo dei nucleopeptidi come conseguenza del wash-out renale e epatico: tali studi risalgono al ventennio '40-60 (19, $22,27,28)$; in seguito è stato dimostrato anche l'effetto del wash-out sugli organi endocrini e sulla loro funzione (29).

Tuttavia, come abbiamo già ricordato, i meccanismi attraverso i quali il trattamento idropinico eserciterebbe un'azione metabolica sono, a tutt'oggi, poco conosciuti: è stato possibile ipotizzare, sulla base dell'osservazione di alcune modificazioni enzimatiche in corso di wash-out con carico idrico $(28,30)$, che si tratti non solo di un effetto fisico ma di un complesso input metabolico, come dimostra il fatto che il carico idrico ha effetti significativi sull'azotemia, sull'urice- 
mia, sull'azoturia e sull'uricuria. Le concentrazioni ematiche di queste due importanti espressioni del catabolismo proteico e purinico tendono a essere più elevate nelle ore successive al carico idrico per poi ridursi parallelamente all'evento azoturico e uricurico in pazienti non affetti da disturbi del metabolismo purinico, da epatopatie o da malattie renali (31). Spiegare questo fenomeno con il solo effetto fisico del wash-out tissutale è semplicistico: è estremamente probabile invece che l'acqua oligominerale eserciti, rispetto all'acqua di fonte, un'azione depurativa legata a uno stimolo metabolico che essa è in grado di esercitare sui parenchimi.

Il nostró studio ha verificato sperimentalmente i dati emersi dai lavori citati: abbiamo voluto che la ricerca fosse semplice, ma al contempo ben mirata e controllata e ci siamo avvalsi, almeno in parte, dei protocolli sperimentali utilizzati in modo similare dagli autori russi sopracitati (anche perché le acque da loro sperimentate non differiscono nella composizione chimica dall'acqua oligominerale Rocchetta).

I nostri dati hanno evidenziato come non esistano significative differenze nella quantità di urina escreta nelle quattro ore fra i due gruppi esaminati: ambedue hanno eliminato una quantità di urina pari a quella che ci si poteva aspettare in individui sani dopo un carico idrico di $20 \mathrm{ml} / \mathrm{kg}$ di una qualsiasi acqua effettuato nell'arco di 30 minuti. Ciò non ci permette certo di trarre alcuna conclusione sull'effetto diuretico che potrebbe essere invece evidenziato con somministrazioni ed osservazioni prolungate.

Sono invece, a nostro parere, di notevole rilievo i dati emersi dalla nostra osservazione relativi alla maggiore escrezione di acido urico, urea e creatinina con relative clearances significativamente più elevate, osservate nel gruppo trattato con acqua Rocchetta rispetto al gruppo di controllo. Questo dato saliente può essere solo interpretato con una particolare capacità dell'acqua Rocchetta, rispetto a quella utilizzata come controllo, di favorire l'escrezione di quelle sostanze in tempi quanto mai brevi. Le due acque sperimentate presentano differenze nella composizione chimica specie riguardo al contenuto in $\mathrm{HCO}_{3}$ e Na più elevati nell'acqua di controllo e alla carenza di diversi elementi in traccia.

È nota da tempo ed è motivo di grande interesse l'associazione dell'iperuricemia con l'ipertensione, l'aterosclerosi $(32,33)$ e l'obesità $(34)$ oltre che con i ben noti quadri di patologia articolare (35) e di nefropatia uratica (36); l'insorgenza e la gravità di questi quadri patologici è correlata all'entità e alla durata dell'iperuricemia (37).

Il $90 \%$ delle iperuricemie non hanno causa nota (forme primitive): per motivi oscuri, l'acido urico sovrasatura il volume extracellulare (uricemia $>7 \mathrm{mg} / \mathrm{dl}$ ) e tende, favorito da altri fattori quali la temperatura e il $\mathrm{pH}$, a precipitare in forma di cristalli nei tessuti coinvolgendo il sistema immunocompetente con conseguente istituzione di quadri flogistici tendenti alla sclerosi, i quali minano la struttura e compromettono la funzione degli organi colpiti.

È opinione diffusa che nelle iperuricemie primitive si ponga in essere un'iperproduzione dell'acido urico (38) associata a fattori vari.

E opportuno ricordare che l'acido urico viene liberamente filtrato dal glomerulo e completamente riassorbito dal primo tratto del tubulo prossimale: la fisiologica uricuria è il risultato dell'escrezione che avviene nel tratto più distale del tubulo prossimale.

In accordo con gli autori russi (25), possiamo affermare che l'evento uricurico da noi osservato dopo carico idrico con acqua Rocchetta potrebbe essere il risultato di una modulazione negativa del riassorbimento a opera del tubulo prossimale dell'acido urico, e che anche il dato da noi osservato relativo alla maggiore clearance della creatinina nel "gruppo Rocchetta" sia il risultato dell'incremento dei flussi tubulari tale da causare la secrezione tubulare di una quota di creatinina che si aggiunge a quella filtrata (39).

Secondo questa ipotesi, un liquido ipotonico dopo il rapido assorbimento si distribuirebbe per il $70 \%$ nel volume extracellulare e per il $30 \%$ nel- l'intracellulare: l'espansione del volume extracellulare è limitata (infatti non abbiamo osservato significative variazioni del compenso pressorio dopo carico idrico) ma con un importante effetto diluitivo dell'acqua corporea totale che modula la cosiddetta "triarchia integrata" (sensazione di sete, attività adiuretinica e capacità diluitiva renale) (40) nella direzione di stimolare una diuresi a basso contenuto salino.

Tale evento comporterebbe, come già detto, l'incremento dei flussi tubulari, la limitazione del riassorbimento di acido urico nel tubulo prossimale, del riassorbimento di urea nel tubulo collettore e motiverebbe una certa quota di secrezione tubulare di creatinina.

In realtà siamo portati a pensare che $\mathrm{i}$ risultati di questo studio siano dovuti anche a un'azione metabolica e depurativa di quest'acqua: in sede di idrologia funzionale moderna si tende a valutare a pieno le possibilità d'azione biologica delle sostanze presenti in concentrazione minima e fortemente dissociate nell'acqua. A queste sostanze si tende ad attribuire effetti biocatalizzatori a livello cellulare e subcellulare. Le analisi delle curve di diffrazione con i Raggi X e con spettro di Raman hanno posto in evidenza come le molecole di $\mathrm{H}_{2} \mathrm{O}$ appaiano orientate e coordinate come cristalli liquidi intorno agli ioni: l'attività biologica viene posta in rapporto con l'energia dei campi di soluzione, dissociazione, ionizzazione; le acque oligominerali sono, da questo punto di vista, le acque più ricche di energia (3).

È difficile pensare che l'epatocita assista passivo al wash-out esercitato sul parenchima epatico a opera di un liquido ipotonico, è possibile, invece, che questo sia apportatore al liquido intracellulare oltre che di un volume idrico, di una componente microergica rappresentata dagli elementi fortemente dissociati e che ciò rappresenti un input alle sue funzioni metaboliche.

Di fatto le attività ossidasiche (comprese quelle della xantino-ossidasi), che tanta importanza hanno nell'azione detossicante del fegato nei riguardi sia dei tossici endogeni che di 
quelli esogeni, avvengono con maggiore velocità a un $\mathrm{pH}$ più elevato (41): il $\mathrm{pH}$ dell'acqua oligominerale Rocchetta è di 7.9 .

Il wash-out epatico potrebbe in tal modo favorire le fasi ultime del catabolismo purinico: possiamo dunque affermare che il contributo dell'acqua Rocchetta all'escrezione dell'acido urico ne suggerisce l'assunzione nei disturbi del metabolismo purinico caratterizzati da iperuricemia, anche in base al fatto che i reni gottosi eliminano in modo insufficiente l'acido urico a diuresi inferiori a $1 \mathrm{ml} / \mathrm{min}$; la terapia idropinica con acqua oligominerale Rocchetta potrebbe rappresentare un valido ausilio alle cure farmacologiche di questi quadri patologici.

Altro dato interessante è che il ciclo dell'urea avviene con una velocità dipendente dalla disponibilità di acqua: l'uomo sceglie l'urea come mezzo di eliminazione dell'azoto e ciò avviene con un costo rappresentato da una certa spesa idrica; esistono animali che hanno minore disponibilità idrica rispetto all'uomo e, dovendo difendere il proprio patrimonio idrico con maggiore efficienza, scelgono come mezzo d'eliminazione dell'azoto un'altra molecola che gli costa una minore spesa idrica e cioè l'acido urico (animali uricotelici) (42).

Il contributo volumetrico del washout epatico al liquido intracellulare potrebbe, perciò, stimolare il ciclo dell'urea.

L'effetto certo del wash-out epatico resta comunque quello meccanico, già documentato da Mian nel 1950 (31): il wash-out veicola acido urico e urea alle successive fasi del loro ciclo metabolico.

A nostro parere non crediamo si possa speculare su questi risultati se non utilizzarli come stimolo alla ulteriore ricerca di quei fini meccanismi d'azione con i quali alcune acque minerali esercitano azioni benefiche sulle funzioni organiche: l'acqua Rocchetta ha comunque dimostrato di esercitare effetti metabolici e depurativi che ne suggeriscono l'assunzione continuativa nell'ambito di una corretta alimentazione.

\section{BIBLIOGRAFIA}

1. Bocconi G. Lezioni di Idrologia Medica. Milano, 1972.

2. Messini M. Trattato di Idroclimatologia Clinica. Bologna, 1950.

3. Comel M. Riepiloghi di Idrologia Medica. Pisa: Ed Omnia Medica $1972 ; 86-100$.

4. Di Paolo N, Capotondo L. Fons Vitae. L'acqua oligominerale Rocchetta nella storia e nella terapia. Pacini Editore 1997; 161.

5. Sarti N. Normativa sulle acque minerali naturali. In: Di Paolo N, Baggio B, Gaggiotti E, eds. Litiasi Renale. Cosenza: Editoriale Bios 1998; 67-71.

6. Di Paolo N, Gaggiotti E. Le acque minerali e la calcolosi renale. In: Di Paolo N, Baggio B, Gaggiotti E, eds. Litiasi Renale. Cosenza: Editoriale Bios 1998; 89-98.

7. Rose A. The medical management of renal stone disease. In: Cameron $\mathrm{S}$ et al, eds. Oxford Texbook of Clinical Nephrology. Ed Oxford Med Pub 1992; 1853.

8. Frank M, Vries A. Prevention of Urolithiasis. Archives of enviromental Health 1966; 13: 625-30.

9. Frank M, Vries A, Atsmon A, Lazebnik J, Kochova S. Epidemiological investigation of urolithiasis in Israel. J Urol 1959; 81: 497-82.

10. Borghi L, Meschi T, Amato F, Briganti A, Novarini A. Urinary volume, water and recurrences in idiopathic calcium nephrolithiasis: a 5year randomized prospective study. J Urol 1996; 155: 839-43.

11. Consensus Conference: Prevention and Treatment of kidney stones. JAMA 1988; 260: 977-81.

12. Ackermann D, Baumann JM, Futterlieb A, Zingg EJ. Influence of calcium content in mineral water on chemistry and cristallization conditions in urine of calcium stone formers. European Urology 1988; 14: 305-8.
13. Pak C. Urolithiasis. In: Schrier RW and Gottschalk CW, eds. Diseases of the kidney. $5^{\mathrm{a}}$ Ed Boston: Little, Brown and C 1993; 25.

14. Smith BL. The pathogenesis and medical treatment of urolithiasis. Sem Nephrol 1990; 10: 31-52.

15. Elliot JP, Gordon JO, Evans JW, Platt L. A stone season. A 10 year retrospective study of 768 surgical stone cases with respect to seasonal variation. J Urol 1975; 4 114: 574-7.

16. Borghi L, Meschi T, Amato F, Novarini A, Romanelli A, Cigala F. Hot occupation and nephrolithiasis. J Urol 1993; 150: 1757-60.

17. Ross JA, Kirkland PES. Behaviour of the human ureter in health and disease. Edimburgo: Churchill Livigstone 1972; 24-32.

18. Agostini G. Alcune osservazioni sulla diuresi da acque oligominerali. In: Di Paolo N, Baggio B, Gaggiotti E, eds Litiasi Renale. Cosenza: Editoriale Bios 2000; 109-18.

19. Agostini G. Reperti funzionali in corso di trattamento idropinico con un'acqua oligominerale. Atti del $48^{\circ}$ Congresso Nazionale dell'Associazione Medica Italiana di Idroclimatologia, Talassologia e Terapia Fisica. Chianciano Terme 1983; 221-33.

20. Ferruzzi M. Contributi al trattamento idropinico oligometallico nella gravidanza. Anthol Medica Santoriana, 1967; Fasc 83.

21. Fiaschi E. L'atteggiamento funzionale del rene normale nella prova di Volhard con acqua oligominerale S. Carlo. Anthol Hydrol, 1952; 1: 27-32.

22. Ghittoni S. Comportamenti di alcune costanti ematiche ed urinarie in corso di trattamento idropinico oligominerale. Anthol Medica Santoriana, 1962; Fasc 64.

23. Di Paolo N. Semeiotica del rene e delle vie urinarie. Milano: Wichtig 1995; 143-70.

24. Dudchenko MA, Sereda LI, Filiuk AP. Effect of Chelkar mineral 
water on the water-salt equilibrium and the function of the kidney. Vopr Kurortol Fizioter Lech Fiz Cult. Russian Mar 1986; 1, 2: 48-51.

25. Mozhaeva IV, Logunova LV, Dzjojev IG, Pronina MN. Mechanism of action of Tib-2 mineral water from North Ossetia on kidney function. Vopr Kurortol Fizioter Lech Fiz Cult. Russian Jul 1984; 1: 47-9.

26. Pronina NN, Logunova LV, Dzjojev IG et al. Effect of mineral water Tib-2 on renal function. Vopr Kurortol Fizioter Lech Fiz Cult. Russian Mar 1986; 1, 2: 48-51.

27. Mecoli V. Azione delle cure idropiniche con un'acqua oligominerale sulla eliminazione urinaria dei corpi purinici, del fosforo, dell'acido ossalico e del magnesio. Rivista d'Idroclimatologia e Talassoterapia, 1940; 7 .

28. Biagini E. Orientamenti funzionali nella terapia delle dermatosi professionali. Anthol De Medicina Externa, 1955; 40: 30-4.

29. Mian EU. Enzimi del siero e carico idropinico oligometallico. Ed Santoriana, 1963.

30. Ferruzzi M. L'eliminazione urinaria dei 17-chetosteroidi e dei 17-idrossicorticosteroidi in donne in climaterio sottoposte a trattamento idropinico oligominerale. Folia Hydrol et Ecologica. Pars Prima, 1969.

31. Mian EU, Guidi IF. Osservazioni nel corso di terapia idropinica di dermatosi varie con acqua S. Carlo. Acta Medica, Santoriana, 1950.

32. Gibson $\mathrm{T}$ et al. Hypertension, renal function and gout. Postgrad Med J 1979; 55: 21.

33. Fessel WJ. Hyperuricemia in health and disease. Semin Arthritis Rheum 1972; 1: 275.

34. Fessel WJ et al. Correlates and consequences of asymptomatic hyperuricemia. Arch Intern Med 1973; 132: 144.

35. Dieppe PA. Crystal deposition and inflammation. Q J Med 1984; 3: 309.
36. Weeden RR, Batumen V. Tubulointerstitial nephritis induced by heavy metals and metabolic disturbances. In: Cotran RS et al, eds. Tubulointerstitial Nephropathies. Contemporary Issues in Nephrology. New York: Churchill Livingstone 1983; 10: 212.

37. Di Paolo N, Capotondo L. Fons Vitae. L'acqua oligominerale Rocchetta nella storia e nella terapia. Pacini Editore: 1997; 169-76.

38. Wyngaarden JB, Kelley WN. Gout. In: Stanbury J et al, eds. The metabolic basis of inherited disease. 5th ed. New York: McGraw-Hill $1983 ; 1043$.

39. Trincheri A. Caratteristiche della diuresi indotta da un carico acuto di acqua oligominerale Rocchetta. Atti del Congresso dell'Associazione Urologi Ospedalieri. 1999.

40. Narins RG, Krishna GG. Alterazioni del bilancio idrico. In: Stein JH, ed Medicina Interna. Mosby Year Book Doyma Italia 1995; $4^{\circ}: 2486-9$.

41. Loscalzo B. L'attività antitossica epatobiliare: possibilità e limiti della crenoterapia. Atti del $48^{\circ}$ Congresso Nazionale dell'Associazione Medica Italiana di Idroclimatologia, Talassologia e Terapia Fisica. Chianciano Terme: 1983; 9-26.

42. Porcellati G, Ricci C, Ronca G. Biochimica. Monduzzi Editore: 1989; 323-61. 